\title{
FK506 immunosuppression for submandibular salivary gland allotransplantation in rabbit
}

\author{
Akram Abdo Almansoori ${ }^{1,2,3,4}$, Namuun Khentii ${ }^{1}$, Kyung Won Ju3 ${ }^{3}$, Bongju Kim³, \\ Soung Min Kim ${ }^{1,4}$, Jong-Ho Lee ${ }^{1,3,4,5}$ \\ ${ }^{I}$ Department of Oral and Maxillofacial Surgery, School of Dentistry, Seoul National University, Seoul, Korea, \\ ${ }^{2}$ Department of Oral and Maxillofacial Surgery, Faculty of Dentistry, Sana'a University, Sana'a, Yemen, \\ ${ }^{3}$ Clinical Translational Research Center for Dental Science, Seoul National University Dental Hospital, \\ ${ }^{4}$ Dental Research Institute, College of Dentistry, Seoul National University, \\ ${ }^{5}$ Oral Cancer Center, Seoul National University Dental Hospital, Seoul, Korea
}

\begin{abstract}
J Korean Assoc Oral Maxillofac Surg 2020;46:197-203)
Objectives: We compared the outcomes of two different doses of FK506 (tacrolimus) for immunosuppression in submandibular salivary gland (SMG) allotransplantation.

Materials and Methods: Three SMG allotransplantation groups were established ( $\mathrm{n}=6$ per group) as follows: allograft rejection control (Allo-Ctrl), low dose $(0.08 \mathrm{mg} / \mathrm{kg}$ ) of FK506 (FK506-L), and high dose $(0.16 \mathrm{mg} / \mathrm{kg})$ of FK506 (FK506-H). Allograft survival and rejection were assessed by clinical observation, interleukin-2 levels as determined by enzyme-linked immunosorbent assay, blood sampling for complete blood count (CBC), and histological evaluation.

Results: Body weight and anorexia were higher in the FK506-H group but without a significant difference compared with the FK506-L population. CBC revealed a non-significantly reduced number of changes in the FK506-L group. Four glands in the FK506-H group and two glands in the FK506L group were viable and functioning post-transplantation.

Conclusion: The survival rate of allotransplanted glands was higher in conjunction with the high dose of $0.16 \mathrm{mg} / \mathrm{kg}$ of FK506, with no major difference in the side-effect profile when compared with the low dose of $0.08 \mathrm{mg} / \mathrm{kg}$ short-term outcomes.
\end{abstract}

Key words: Submandibular salivary gland, Allotransplantation, Immunosuppression, FK506, Tacrolimus [paper submitted 2019. 10. 7 / revised 2019. 12. 12 / accepted 2019. 12. 17]

\section{Introduction}

Allotransplantation of submandibular salivary glands (SMGs) may provide an alternative treatment option for managing xerostomia, or for cases where autologous SMG transplantation is not feasible for managing severe xerophthalmia.

Challenges in allotransplantation include finding a less toxic immunosuppression method. Recently, SMG allotransplan-

\footnotetext{
Jong-Ho Lee

Department of Oral and Maxillofacial Surgery, School of Dentistry, Seoul National University, 101 Daehak-ro, Jongno-gu, Seoul 03080, Korea

TEL: +82-2-2072-2630 FAX: +82-2-766-4948

E-mail:leejongh@snu.ac.kr

ORCID: https://orcid.org/0000-0002-8843-545X

(c) This is an open-access article distributed under the terms of the Creative Commons Attribution Non-Commercial License (http://creativecommons.org/ licenses/by-nc/4.0/), which permits unrestricted non-commercial use, distribution, and reproduction in any medium, provided the original work is properly cited. Copyright (C) 2020 The Korean Association of Oral and Maxillofacial Surgeons. All rights reserved.
}

tation was incorporated in the treatment of severe xerophthalmia in two patients ${ }^{1}$. Both patients presented with complete functional loss of their SMGs and lacrimal glands due to the onset of graft-versus-host disease (GvHD) following stem cell transplantation. The SMG allotransplantations in these cases were performed without immunosuppression because both patients had complete donor chimerism following stem cell transplantation. Postoperative clinical assessments of the patients revealed primary success of the allotransplanted glands with an improvement in ocular surface lubrication and a reduction in inflammatory findings. Nevertheless, long-term sialoscintigraphy follow-up revealed a lower tracer activity than expected and a decreased level of secretion of saliva and tears. As such, further treatment with initial and minimal immunosuppressive therapy was recommended ${ }^{1}$.

The immunosuppressant FK506 (tacrolimus) is a powerful and selective anti-T-lymphocyte agent. It has a similar action 
mechanism to cyclosporine but is more potent ${ }^{2}$. It is a reliable candidate for immunosuppression in SMG transplantation because of its potency and ability to enhance nerve regeneration, which could boost the spontaneous autonomic innervations of transplanted glands ${ }^{3,4}$.

Two different FK506 doses were considered for our planned SMG allotransplantation based on a rabbit animal model. A dose of $0.08 \mathrm{mg} / \mathrm{kg}$ FK506 was effective and less toxic for rabbit lower limb allotransplantation procedures and a $0.16 \mathrm{mg} / \mathrm{kg}$ FK506 dose was applied for SMG allotransplantation in $\operatorname{dog} s^{5,6}$. Given this information, we conducted the present study to compare the outcomes of two doses $(0.08$ $\mathrm{mg} / \mathrm{kg}$ and $0.16 \mathrm{mg} / \mathrm{kg}$ ) of FK506 in SMG allotransplantation.

\section{Materials and Methods}

\section{Animals and groups}

This study was approved by the Institutional Animal Care and Use Committee (SNU-160720-6-2). SMG allotransplantations were completed in the right side of New Zealand white female rabbits (donors, $\mathrm{n}=18$ ) and New Zealand white male rabbits (recipients, $\mathrm{n}=18$ ) weighing 3 to $4 \mathrm{~kg}$. All animals were nonrelated and were observed for two weeks before the terminal experiment. For this study, the rabbits were randomly divided into the following three groups, each containing six animals: (1) allograft rejection control group (Allo-Ctrl), (2) maintenance immunosuppression with lowdose FK506 (0.08 mg/kg) (FK506-L) group, and (3) maintenance immunosuppression with high-dose FK506 (0.16 mg/ kg) (FK506-H) group.

\section{Surgical procedures}

The animal surgeries and experimental procedures were conducted in a manner previously described for SMG replantation ${ }^{7}$. Briefly, general anesthesia was maintained with isoflurane $(1.5 \%-2.5 \%)$ via a rabbit supraglottic V-gel tube (Docsinnovent, London, UK). Dissection was performed to identify the linguofacial vein and common carotid artery. The SMG was then separated and all of the common carotid artery branches were transected and ligated while preserving the facial artery and its glandular branch. Similarly, the lingual and facial veins were ligated while preserving the linguofacial vein trunk and the glandular vein. Wharton's duct was identified and a silicone tube measuring $0.5 \mathrm{~mm}$ in di- ameter (Beaver-Visitec, Waltham, MA, USA) was introduced into the duct. The receiver rabbit was then anesthetized and its right SMG was removed. The vessels were prepared in the same manner as for the donor. The vascular pedicles were connected by vascular microanastomoses of the linguofacial vein and common carotid artery to their corresponding proximal ends in an end-to-end fashion using 10-0 nylon (Ethicon, Somerville, NJ, USA) under a surgical microscope (Carl Zeiss, Oberkochen, Germany). The ischemia time was calculated form the time of SMG vessel detachment in the donor site to the time of blood reperfusion in the recipient site. Blood reperfusion was checked by laser Doppler (Perimed AB, Jarfalla, Sweden). Saliva flow was reconfirmed and the tube cannulated into the donor Wharton's duct was connected to the remaining distal Wharton's duct of the receiver. The wound was then irrigated gently with normal saline and closed using 4-0 Vicryl (Ethicon) for the platysma-fascia layer and 4-0 Dafilon (B. Braun, Melsungen, Germany) for the skin. A Penrose drain was placed for three days. Postoperatively, levofloxacin $(5 \mathrm{mg} / \mathrm{kg}$ ) was administered twice daily for three days, cephazolin $20 \mathrm{mg} / \mathrm{kg}$ once daily for five days, and tramadol ( $5 \mathrm{mg} / \mathrm{kg}$ ) was administered twice daily for three days. Additionally, dextrose 5\% (20 mL) and $0.9 \%$ normal saline $(70 \mathrm{~mL})$ were adjunctively supplied on a daily basis for three days. Assisted oral feeding with a Critical Care Formula (Oxbow Animal Health, Omaha, NE, USA) was introduced beginning on the third postoperative day in cases where the rabbit did not resume feeding spontaneously.(Fig. 1)

\section{Maintenance immunosuppression}

FK506 was used for immunosuppression. As previously indicated, a dose of $0.08 \mathrm{mg} / \mathrm{kg}$ was administered to the FK506-L group, while a dose of $0.16 \mathrm{mg} / \mathrm{kg}$ was administered to the FK506-H group, based on the work of Giessler et al. ${ }^{5}$ and Tanaka Isomura et al. ${ }^{6}$. Starting one day before transplantation, the drug was given intravenously, twice a day, as bolus injections over 90 minutes without monitoring of blood tacrolimus levels ${ }^{8,9}$.

\section{Health condition and rejection surveillance}

1) Clinical observations

The glands were gently palpated post-transplantation to detect any hardness, which is a sign of immunorejection in this context $^{10}$. Body weight variations were calculated based on 

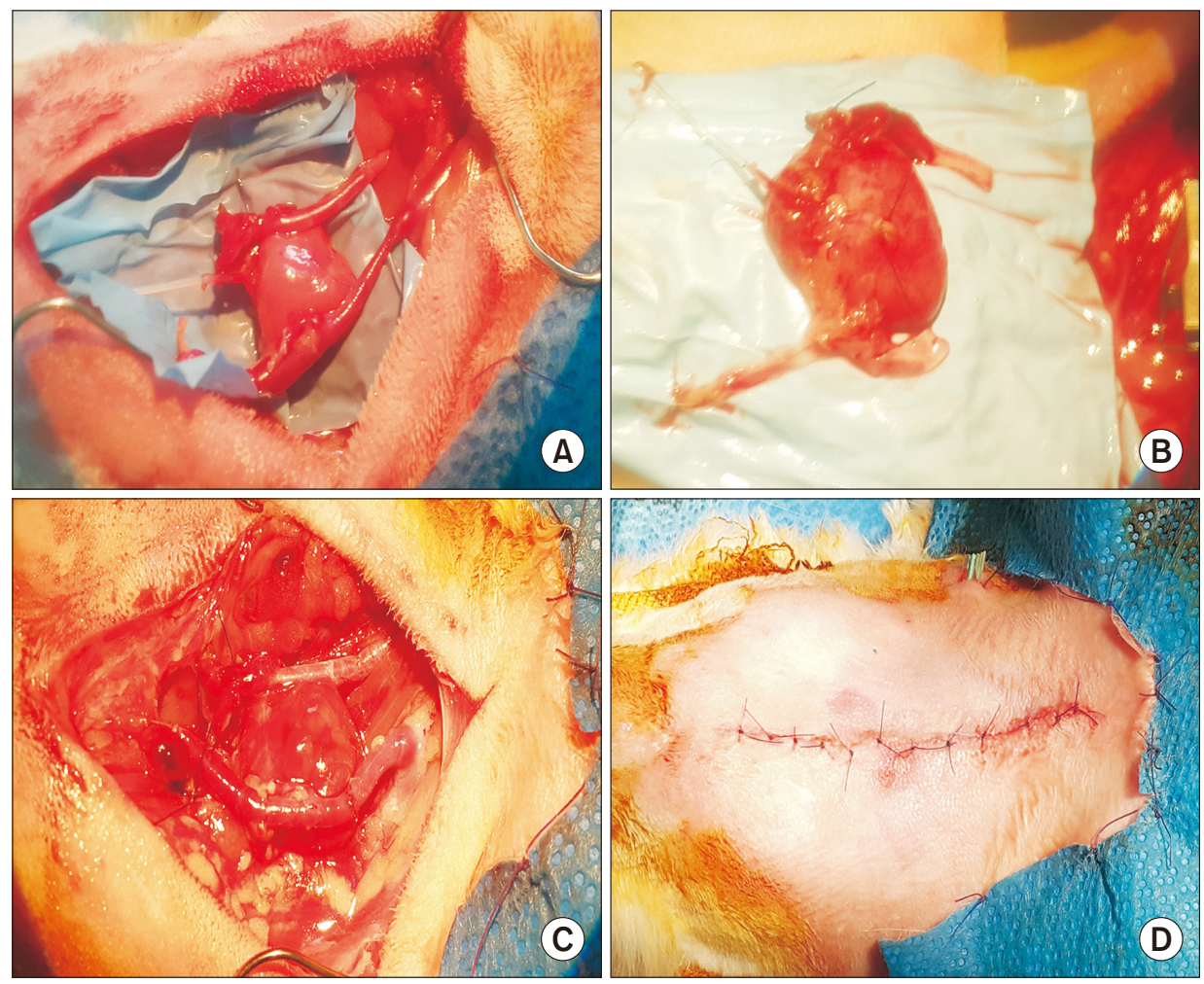

Fig. 1. Intraoperative clinical photographs for submandibular salivary gland (SMG) allotransplantation. A. Complete separation of the SMG in the donor rabbit with an intact linguofacial vein, common carotid artery, and silicon tube connected to Wharton's duct. B. Harvested SMG over the receiver chin with proper orientation of the vessels. C. After vascular anastomosis and connection to the remaining distal Wharton's duct of the receiver. D. After skin suturing and drain placement.

Akram Abdo Almansoori et al: FK506 immunosuppression for submandibular salivary gland allotransplantation in rabbit. J Korean Assoc Oral Maxillofac Surg 2020 the weights recorded on the days of transplantation and sacrifice. Anorexia and spontaneous feeding of the animals were noted as well.

\section{2) Interleukin-2 (IL-2) detection}

Serum samples for measuring IL-2 with enzyme-linked immunosorbent assay (ELISA) (Cat. \#E-EL-RB-1217; Elabscience Biotechnology, Houston, TX, USA) were taken 11 days after allotransplantation; $100 \mu \mathrm{L}$ (duplicate) of each sample and a standard per well were added to the ELISA plate, and the ELISA plate was then incubated for $90 \mathrm{~min}-$ utes at $37^{\circ} \mathrm{C}$. Liquids were removed and a $100 \mu \mathrm{L} /$ well of biotinylated detection antibody was added and incubated for 60 minutes at $37^{\circ} \mathrm{C}$. The wells were then washed with wash buffer three times and incubated for 30 minutes at $37^{\circ} \mathrm{C}$ following the addition of Horseradish peroxidase-conjugate working solution. The wells were washed an additional five times and incubated for 23 minutes at $37^{\circ} \mathrm{C}$ after the addition of a $90 \mu \mathrm{L} /$ well of substrate reagent. Stop solution was subsequently added to each well and the optical density of each well was determined using a microplate reader set to $450 \mathrm{~nm}$.

\section{3) Blood sampling}

For the overall evaluation of the animals' general health, blood samples were obtained on the day of sacrifice for com- plete blood count (CBC) analysis, including red blood cell count, white blood cell count, and hemoglobin ( $\mathrm{Hgb})$ and hematocrit (HCT) levels.

\section{4) Saliva secretion}

At the end of the two week experimental interval, the rabbits were anesthetized and injected with pilocarpine $(1.5 \mathrm{mg} /$ $\mathrm{kg})^{6,11}$ to stimulate saliva secretion.

\section{5) Histological evaluation}

Glands were harvested and fixed in 10\% formaldehyde before being divided into equal groups of specimens, embedded in paraffin, sectioned, and stained with H\&E for histological evaluation. The glandular tissue was evaluated in terms of the percentage of parenchymal degeneration, acinar atrophy, and inflammatory cell infiltration. Based on the work of Kumar et $\mathrm{al}^{12}$, the surviving glands were deemed to be those with nearnormal histological glandular tissue or in which more than $50 \%$ of the glandular tissue was preserved.

\section{Statistical analysis}

Data analysis was conducted using the IBM SPSS Statistics (ver. 23.0; IBM Corp., Armonk, NY, USA). All data are presented as the mean with standard error of the mean. The asso- 
ciation of nominal variables was tested using the chi-squared test. Nonparametric data comparisons were conducted with the Mann-Whitney U-test. For parameter data, the analysis of variance test followed by the post-hoc least significant difference test was used to compare data between different groups. A $P$-value less than 0.05 was considered to be statistically significant.

\section{Results}

\section{Clinical findings}

Intraoperatively, all glands other than one gland in the Allo-Ctrl group showed patency of anastomosed vessels and salivary flow. The ischemia time ranged between 92 and 106 minutes, with no significant differences observed among the groups. Within six to seven days, four glands of the Allo-Ctrl group and one gland of the FK506-L group were completely hard. One rabbit in the FK506-H group died on the sixth day and its allotransplanted SMG was found to be necrotic. Overall, body weight loss was higher in the FK506-H group (mean, $0.49 \pm 0.09 \mathrm{~kg}$ ) than in the FK506-L group (mean, $0.44 \pm 0.10$ $\mathrm{kg}$ ) but without any significant difference. Anorexia was common, as seen in three rabbits in the FK506-L (50.0\%) and four rabbits in the FK506-H (66.6\%) groups.(Table 1)

\section{Low interleukin-2 level}

The expression level of IL-2 in the serum of all animals was undetectable.

\section{Blood sampling results}

The $\mathrm{CBC}$ revealed lower Hgb and HCT levels in the FK506-H group than in the F506-L group, without a statistically significant difference. The percentage of lymphocytes was similar in the two FK506 groups but higher in the control group, while the percentage of monocytes was higher in the

Table 1. Body weight change and anorexia percentages

\begin{tabular}{lccc}
\hline \multicolumn{1}{c}{ Variable } & $\begin{array}{c}\text { FK506-H } \\
\text { group }\end{array}$ & $\begin{array}{c}\text { FK506-L } \\
\text { group }\end{array}$ & Total \\
\hline Body weight loss (kg) & $0.49 \pm 0.09$ & $0.44 \pm 0.10$ & $0.46 \pm 0.09$ \\
Anorexia (\%) & 66.6 & 50.0 & 58.3 \\
\hline (FK506-H: high-dose & FK506 $[0.16 \mathrm{mg} / \mathrm{kg}]$, FK506-L: low-dose \\
FK506 [0.08 mg/kg]) & & \\
Values are presented as mean \pm standard error of the mean or \%. \\
Akram Abdo Almansoori et al: FK506 immunosuppression for submandibular salivary \\
gland allotransplantation in rabbit. J Korean Assoc Oral Maxillofac Surg 2020
\end{tabular}

FK506-L group than in the FK506-H group, although the result was not statistically significant. No major differences for the other hematological parameters were found between the groups.(Table 2)

\section{Saliva secretion}

Saliva was found in three of the FK506-H glands and in one gland in the FK506-L group.

\section{Histological changes in the allotransplanted glands}

Nearly normal glandular tissue structure was predominant in one gland in the FK506-H group. Partial loss of the parenchyma (not exceeding 50\%) and an increase in fibrous tissue were findings in three glands in the FK506-H group and two glands in the FK506-L group. A loss of the majority of the parenchyma and an abundance of fibrous tissue were seen in one gland in the FK506-H group. Complete rejection was characterized by the complete loss of the parenchyma, except for a few remnant ducts with marked inflammatory cell infiltration, as was observed in five glands in the AlloCtrl group and three in the FK506-L group. The necrosis of allotransplanted glands due to failed blood perfusion was characterized by acini fragmentation with autolysis, as seen in one gland in each of the Allo-Ctrl, FK506L, and FK506-H groups.(Fig. 2)

\section{Discussion}

Allotransplanted SMG can be rejected within the first few days post-transplantation. To, the best of our knowledge, Wang et al. ${ }^{13}$ is the first group to have conducted vascularized SMG allotransplantation. In their study, SMG allotransplantation was performed in 40 rabbits without immunosuppres-

Table 2. Complete blood count changes within the different groups

\begin{tabular}{lrrrr}
\hline Variable & \multicolumn{1}{c}{$\begin{array}{c}\text { Allo-Ctrl } \\
\text { group }\end{array}$} & \multicolumn{1}{c}{$\begin{array}{c}\text { FK506-L } \\
\text { group }\end{array}$} & \multicolumn{1}{c}{$\begin{array}{c}\text { FK506-H } \\
\text { group }\end{array}$} & \multicolumn{1}{c}{ Total } \\
\hline LY $(\%)$ & $63.75 \pm 8.63$ & $47.63 \pm 10.18$ & $49.73 \pm 6.14$ & $53.70 \pm 4.91$ \\
MO (\%) & $3.68 \pm 1.26$ & $3.90 \pm 1.37$ & $1.93 \pm 0.44$ & $3.17 \pm 0.63$ \\
Hgb (\%) & $11.80 \pm 0.28$ & $12.30 \pm 1.14$ & $10.75 \pm 1.54$ & $11.61 \pm 0.62$ \\
HCT (fl) & $35.38 \pm 0.97$ & $37.36 \pm 3.35$ & $32.60 \pm 4.98$ & $35.11 \pm 1.96$ \\
\hline
\end{tabular}

(Allo-Ctrl: allograft rejection control, FK506-L: low-dose FK506 [0.08 $\mathrm{mg} / \mathrm{kg}$ ], FK506-H: high-dose FK506 [0.16 mg/kg], LY: lymphocyte, MO: monocyte, Hgb: hemoglobin, HCT: hematocrit)

Values are presented as mean \pm standard error of the mean.

Akram Abdo Almansoori et al: FK506 immunosuppression for submandibular salivary gland allotransplantation in rabbit. J Korean Assoc Oral Maxillofac Surg 2020 

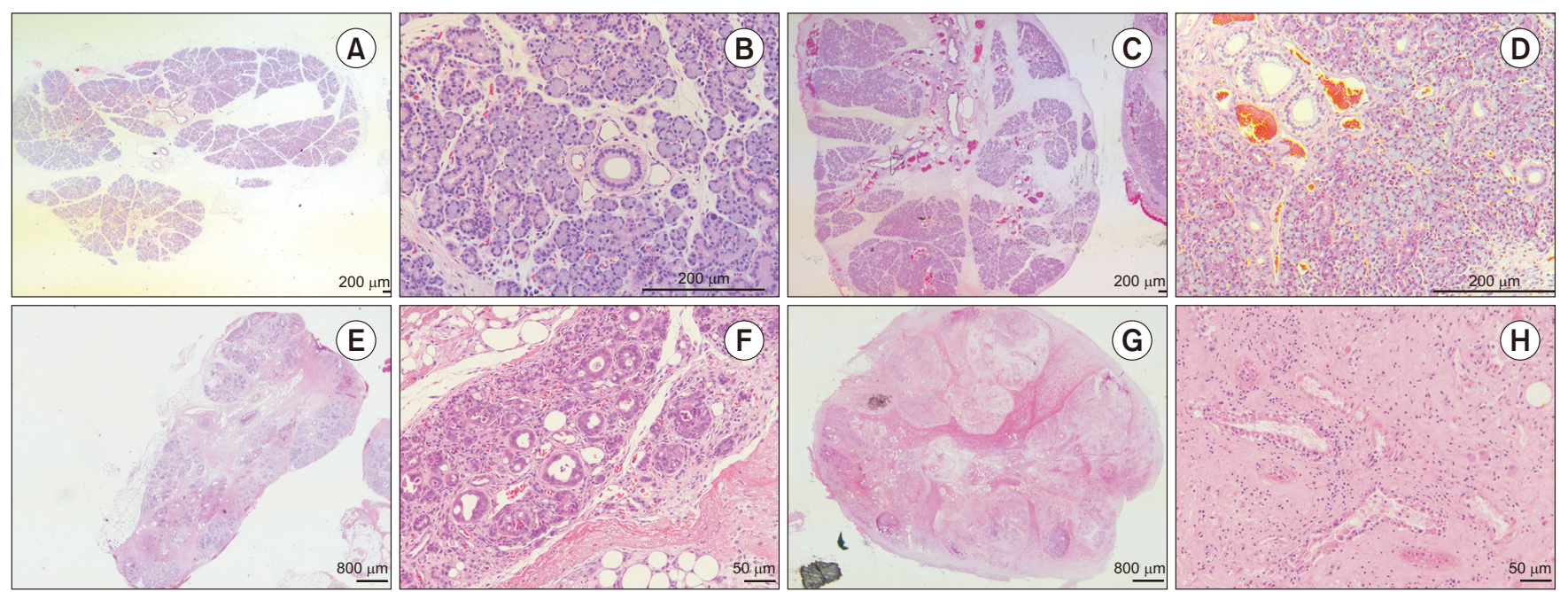

Fig. 2. Photomicrographs of allotransplanted glands as shown by H\&E staining. A. Histological features of an allotransplanted gland with nearly normal glandular parenchyma structure (magnification, $\times 1.25$; scale bar $=200 \mu \mathrm{m}$ ). B. The same allotransplanted gland (magnification, $\times 20$; scale bar $=200 \mu \mathrm{m})$. C. Histological features of an allotransplanted gland with partial loss of the parenchyma (magnification, $\times 1.25$; scale bar=200 $\mu \mathrm{m}$ ). D. The same allotransplanted gland (magnification, $\times 20$; scale bar=200 $\mu \mathrm{m})$. E. Histological features of an allotransplanted gland with loss of the majority of the parenchyma and an abundance of fibrous tissue (magnification, $\times 1.25 ; \mathrm{scale}$ bar=800 $\mu \mathrm{m}$ ). $\mathrm{F}$. The same allotransplanted gland (magnification, $\times 20$; scale bar=50 $\mu \mathrm{m}$ ). G. Histological features of a rejected allotransplanted gland with acini degeneration and marked inflammatory cells infiltration (magnification, $\times 1.25$; scale bar $=800 \mu \mathrm{m}$ ). $\mathrm{H}$. The same rejected gland (magnification, $\times 20$; scale bar $=50 \mu \mathrm{m})$.

Akram Abdo Almansoori et al: FK506 immunosuppression for submandibular salivary gland allotransplantation in rabbit. J Korean Assoc Oral Maxillofac Surg 2020

sion. The glands survived for five days and were rejected after seven days. The authors concluded that, without using immunosuppressants, acute rejection occurs following the allotransplantation of SMGs with blood vessel anastomosis.

Different animal models and immunosuppressants have been used for SMG allotransplantation. Ge et al..$^{10}$ conducted SMG allotransplantation with immunosuppression in swine animal models using high and low doses of cyclosporine, methylprednisolone, and azathioprine. In their study, SMGs transplanted into control subjects that were not given immunosuppressants were rejected within seven days. The rejected glands were hard to the touch and showed histological changes. In the low-dose immunosuppressant group, allografts were rejected on Days 15, 17, 19, and 20 after the operation, while, in the high-dose immunosuppressant group, the survival time of the allografts was considerably prolonged, with three allografts rejected on Days 35, 48, and 60 and another two allografts surviving for 100 days without signs of rejection. Later, Tanaka Isomura et al. ${ }^{6}$ reported on SMG allotransplantations in beagle dogs that were successful for 12 weeks, using $0.16 \mathrm{mg} / \mathrm{kg}$ of tacrolimus as an immunosuppressant.

The first human allogeneic SMG transplantations were conducted in two patients with complete chimerism following stem cell transplantation, with primarily successful results. However, long-term follow-up with sialoscintigraphy showed a decrease in the tracer uptake, and the authors recommended minimal and initial immunosuppression with the allotransplantation.

FK506 was selected for the present study owing to its superior potency in comparison with cyclosporine. In this study, the high and low $(0.08 \mathrm{mg} / \mathrm{kg}$ and $0.16 \mathrm{mg} / \mathrm{kg})$ doses that were administered resulted in the survival of four and two glands, respectively, out of six in each group. The side effects of body weight loss, anorexia, and changes in blood parameters revealed in the animals during this short-term study tended to be less significant in the FK506-L group than in the FK506-H group but the results were not statistically significant. The rabbits showed anorexia, particularly in the first three to five days. Later, almost half of them resumed feeding spontaneously, and the rest were compliant with assisted feeding, as needed. The amelioration of anorexia was particularly noticeable after ceasing the administration of antibiotics and reducing the surgical wound pain level. One rabbit in the FK506-H group died on the sixth day post-transplantation as a complication of diarrhea. Blood analysis showed non-significantly lower Hgb and HCT levels in the FK506-H group. However, these levels were still within the normal ranges and such an effect could be attributed to the nutrition status of the rabbits, which could be related to the FK506 dosage and anorexia level ${ }^{14,15}$. Monocytes were non-significantly higher 
in the FK506-L group and have been reported to be closely related to acute allograft dysfunction ${ }^{16,17}$.

Of note, some patients with Sjögren's syndrome or GvHD may already take systemic FK506 to treat underlying causes of their disease or to alleviate dry eye symptoms ${ }^{18,19}$. Furthermore, FK506 has neuroprotective and neuroregenerative properties for neurodegenerative disorders and peripheral nerve injuries ${ }^{20,21}$. Elsewhere, FK506 has been reported to exert neurotrophic effects on the postganglionic parasympathetic cavernous nerve ${ }^{22}$. Such properties would boost SMG autonomic neural regeneration and subsequently result in a reduced latent saliva production period.

\section{Conclusion}

The survival rate of the allotransplanted glands was higher when using a dose of $0.16 \mathrm{mg} / \mathrm{kg}$ of FK506 in comparison with a dose of $0.08 \mathrm{mg} / \mathrm{kg}$, and with no significant differences in the side effect profile between the two doses. Regardless of dose concentration, the two groups that took FK506 had notable advantages compared to the Allo-Ctrl group. Therefore, the results support the use of FK506 for immunosuppression in salivary gland allotransplantation.

\section{ORCID}

Akram Abdo Almansoori, https://orcid.org/0000-00017134-9195

Namuun Khentii, https://orcid.org/0000-0002-2992-3621

Kyung Won Ju, https://orcid.org/0000-0002-2566-7405

Bongju Kim, https://orcid.org/0000-0001-7309-5977

Soung Min Kim, https://orcid.org/0000-0002-6916-0489

Jong-Ho Lee, https://orcid.org/0000-0002-8843-545X

\section{Authors' Contributions}

A.A.A., N.K., and K.W.J. participated in data collection and wrote the manuscript. J.H.L. and A.A.A. participated in the study design. B.K. performed the statistical analysis. S.M.K. and J.H.L. participated in the study design and coordination and helped to draft the manuscript. All authors read and approved the final manuscript.

\section{Acknowledgements}

This research was supported by a grant of the Korea Health Technology R\&D Project through the Korea Health Industry
Development Institute (KHIDI), funded by the Ministry of Health \& Welfare, Republic of Korea (No. HI15C1535).

\section{Ethics Approval and Consent to Participate}

This study was approved by the Institutional Animal Care and Use Committee (SNU-160720-6-2).

\section{Conflict of Interest}

No potential conflict of interest relevant to this article was reported.

\section{References}

1. Jacobsen HC, Hakim SG, Trenkle T, Nitschke M, Steven P, Sieg P. Allogenic submandibular gland transplantation following hematopoietic stem cell transplantation. J Craniomaxillofac Surg 2013;41:764-9.

2. Wallemacq PE, Reding R. FK506 (tacrolimus), a novel immunosuppressant in organ transplantation: clinical, biomedical, and analytical aspects. Clin Chem 1993;39(11 Pt 1):2219-28.

3. Wang MS, Zeleny-Pooley M, Gold BG. Comparative dosedependence study of FK506 and cyclosporin A on the rate of axonal regeneration in the rat sciatic nerve. J Pharmacol Exp Ther 1997;282:1084-93.

4. Geerling G, Garrett JR, Paterson KL, Sieg P, Collin JR, Carpenter $\mathrm{GH}$, et al. Innervation and secretory function of transplanted human submandibular salivary glands. Transplantation 2008;85:13540.

5. Giessler GA, Gades NM, Friedrich PF, Bishop AT. Severe tacrolimus toxicity in rabbits. Exp Clin Transplant 2007;5:590-5.

6. Tanaka Isomura E, Yoshitomi K, Hamaguchi M, Yamamoto YE, Kogo M. Transplantation of vascularized submandibular gland in dogs. J Oral Maxillofac Surg 2006;64:1561-5.

7. Almansoori AA, Khentii N, Hei WH, Seo N, Lee SH, Kim SM, et al. Rabbit submandibular salivary gland replantation. J Korean Assoc Oral Maxillofac Surg 2017;43:299-304.

8. Snell GI, Ivulich S, Mitchell L, Westall GP, Levvey BJ. Evolution to twice daily bolus intravenous tacrolimus: optimizing efficacy and safety of calcineurin inhibitor delivery early post lung transplant. Ann Transplant 2013;18:399-407.

9. Skeens M, Pai V, Garee A, Termuhlen AM, Bajwa RP, Gross TG, et al. Twice daily i.v. bolus tacrolimus infusion for GVHD prophylaxis in children undergoing stem cell transplantation. Bone Marrow Transplant 2012;47:1415-8.

10. Ge XY, Yu GY, Cai ZG, Mao C. Long-term survival of an allografted submandibular gland in a miniature swine model given immunosuppressant drugs. Br J Oral Maxillofac Surg 2006;44:14651.

11. Brown DM, Quinton RM. The assay of anti-acetylcholine agents for antagonism of pilocarpine-induced salivation in rabbits. Br J Pharmacol Chemother 1957;12:53-60.

12. Kumar PA, Macleod AM, O'Brien BM, Hickey MJ, Knight KR. Microvascular submandibular gland transfer for the management of xerophthalmia; an experimental study. Br J Plast Surg 1990;43:431-6.

13. Wang J, Wang Y, Lu L, Sun C, Shao D. Histology of the submandibular glands in rabbits after allotransplantation. J China Med Univ 2000;29:462-4. 
14. Etim NN, Enyenihi GE, Akpabio U, Offiong EEA. Effects of nutrition on haematology of rabbits: a review. Eur Sci J 2014;10:41324.

15. Maheshwari A, Mishra R, Thuluvath PJ. Post-liver-transplant anemia: etiology and management. Liver Transpl 2004;10:165-73.

16. Girlanda R, Kleiner DE, Duan Z, Ford EA, Wright EC, Mannon $\mathrm{RB}$, et al. Monocyte infiltration and kidney allograft dysfunction during acute rejection. Am J Transplant 2008;8:600-7.

17. Magil AB. Monocytes/macrophages in renal allograft rejection. Transplant Rev (Orlando) 2009;23:199-208.

18. Cordero-Coma M, Anzaar F, Sobrin L, Foster CS. Systemic immunomodulatory therapy in severe dry eye secondary to inflammation. Ocul Immunol Inflamm 2007;15:99-104.

19. Aoki S, Mizote H, Minamoto A, Suzuki M, Mishima HK, Tanaka H. Systemic FK506 improved tear secretion in dry eye associated with chronic graft versus host disease. Br J Ophthalmol 2005;89:243-4.

20. Yeh C, Bowers D, Hadlock TA. Effect of FK506 on functional recovery after facial nerve injury in the rat. Arch Facial Plast Surg
2007;9:333-9.

21. Steiner JP, Hamilton GS, Ross DT, Valentine HL, Guo H, Connolly MA, et al. Neurotrophic immunophilin ligands stimulate structural and functional recovery in neurodegenerative animal models. Proc Natl Acad Sci U S A 1997;94:2019-24.

22. Burnett AL, Becker RE. Immunophilin ligands promote penile neurogenesis and erection recovery after cavernous nerve injury. $\mathrm{J}$ Urol 2004;171:495-500.

How to cite this article: Almansoori AA, Khentii N, Ju KW, Kim B, Kim SM, Lee JH. FK506 immunosuppression for submandibular salivary gland allotransplantation in rabbit. J Korean Assoc Oral Maxillofac Surg 2020;46:197-203. https://doi.org/10.5125/jkaoms.2020.46.3.197 